\title{
Time to recovery from moderate acute malnutrition and its predictors among 6-59 months children targeted for supplementary feeding in Shalla District, West Arsi Zone, Ethiopia,2018.
}

kebede kumsa sadeta ( $\square$ kebedekumsa929@gmail.com )

Madawalabu university

Wondwosen Tekle silassie

Hawassa University

Research article

Keywords: Time to recovery, targeted supplementary feeding, and moderate acute malnutrition.

Posted Date: December 20th, 2019

DOI: https://doi.org/10.21203/rs.2.16368/v2

License: (a) This work is licensed under a Creative Commons Attribution 4.0 International License.

Read Full License 


\section{Abstract}

Background-Globally, acute malnutrition accounts for $>50 \%$ cases of childhood mortality in under 5 children each year. Moderate acute malnutrition treatment would be to reduce child mortality and morbidity. Study done in Ethiopia showed treatment food share, lack of water and other factor suggested to cause lower chance of recovery at end of 16 week as per Ethiopian acute malnutrition treatment guide line. Methods- Retrospective cohort study design was conducted among children aged 6-59 months which were treated in targeted supplementary feeding program . A total of 402 children were selected from eight health posts by systematic random sampling. Data were collected by reviewing registration records and interview from January 1-10/2018. Data were analyzed using SPSS version 20, bivariate and multivariate statistical methods were used to analyze and describe the data and $p<0.05$ was considered as significant. Results-the overall average timely recovery was 15 week (SD , 3.3). Children treated by super cereal were 2.44 times more likely to recover timely than children treated by corn soybean blend (AHR 2.44; 95\% Cl 1.85-3.21). Children that had regular follows up has $48 \%$ (AHR 1.48; 95\% $\mathrm{Cl} 1.09-2.00$ ) more chance of recovery than intermittent treatment follow up, children's from sever food in secured family had $51 \%$ lower chance to recover than moderate food insecured house hold (AHR $0.49 ; 95 \% \mathrm{Cl}$ $0.21-0.11$ ) and, admission MUAC ${ }^{3} 11.6 \mathrm{~cm}$ had 1.18 times higher proportion of recovery than admission MUAC $=11.5 \mathrm{~cm}($ AHR 1.18; 95\% Cl 1.13-1.12). Children who have no transport access has 2 times lower proportion of recovery than who have access (AHR $0.5 ; 95 \% \mathrm{Cl} 0.38-0.64$ ). Conclusion- the mean time to recovery was 15 week which is consistent with the national standard 16 weeks. Type of treatment foods, treatment follow up status, and admission mid upper arm circumference had significant association with time recovery moderate acute malnutrition treatment. Keyword : -Time to recovery, targeted supplementary feeding, and moderate acute malnutrition

\section{Background}

Acute malnutrition continues to be a major global public health problem affecting an estimated 51.5 million children aged less than 5 years and being associated with $12.6 \%$ of all under- 5 child mortality[1]. Globally about 33 million children had MAM. Thus, MAM affects about one in ten children under 5 years in the least developed countries[2, 3].

In Ethiopia $10 \%$ of children 6-59 months of age are wasted, with $70 \%$ of these having MAM, and about $35 \%$ to $57 \%$ of the deaths in fewer than five children are attributable to malnutrition [4].

Moderate acute malnutrition is weight for height indicator between -3 and -2 Z- score standard deviation of the international standard or mid upper arm circumference (MUAC) between $11.5 \mathrm{~cm}$ and $12.5 \mathrm{~cm}$ [7].

There are risk factors for MAM and SAM such as inadequate dietary intake, inappropriate feeding, fetal growth restriction, inadequate sanitation, lack of parental education, family size, incomplete vaccination, poverty, economic, political, and environmental instability and emergency situations. When compared to 
adequately nourished children, children suffering from acute malnutrition have increased risk of mortality, impaired physical and cognitive development, and infections [8].

Acute malnutrition is the major cause for child mortality. A child with MAM is up to 3 times as likely to die as well as well-nourished child. Child with SAM is nine times as likely to die as well-nourished child. For every child suffering from severe acute malnutrition there is eight to 10 suffering for moderate acute malnutrition and therefore absolute mortality is higher for MAM than SAM [9].

Targeted Supplementary Feeding is implemented in food insecure situations, including in emergencies, in order to treat MAM and to prevent children with MAM from becoming severely malnourished (falling into SAM) and usually indicated when MAM and SAM prevalence rates are between $10-14 \%$ or $5-9 \%$ with aggravating circumstances [5].

To tackle this problem government of Ethiopia implemented TSFP activities in food insecure setting. Moderate acute malnourished children screened from community using MUAC, admitted to TSFP, promotive and preventive services such as provision of food ration, vitamin A supplementation and deworming is done according to MAM case management guidelines, and protocols.

Despite standardized management, studies indicated that the child timely recovery for MAM ranges from $49.2 \%$ to $73 \%[2,10]$.

Studies conducted in Jimma by save the children and world food programme Ethiopia showed, only $49.2 \%$ recovered at 6 month follow-up and $47.2 \%$ did not recovered at 3 months of TSF treatments [6].

Though recovery time of MAM is with wide statistical range, no study reported MAM time of recovery of Shalla district, west Arsi zone. Due different factor $12.5 \%$ children failed to achieve their target weight within specified period and a little research is done on this area to explain factor that affect time of recovery in the study area [10].

Some of TSF beneficiary children deteriorate or remain for long time without improvement for different reasons such as intra-household sharing, targeting problem and delay of distribution or other factor. Therefore, this study attempts to investigate time to recovery to moderate acute malnutrition and its predictors among 6-59 months children targeted for supplementary feeding in case of Shalla district Oromia region.

\section{Methods}

\section{Study area and period}

The study was conducted in the WestArsi zone shalla district health posts namely, Aje, S.shalla, Abure, Soni,llaala, funde, Awara, and kubi between May 1, 2017, and August 30, 2017. 
The zone has an estimated total population of 2,542,569 and 529,702 households according to annual performance report of $2017 \mathrm{Gc}$ [27].

According to shalla district health office report a total population of the district is 149,804 , of whom 74,930 were men and 74,874 were women; 7,680 or $5.13 \%$ of its population were urban dwellers in 2017 G.c. and Children aged 6-12 month at risk of developing moderate acute malnutrition is 3318 [15]. Food products commonly produced in the district is corn, potatoes, soybean commonly used for consumption and market purpose which is not enough throughout the year. To alleviate problems related to food insecurity there is targeted and blanket food supply program in the district. There are 8 government health centers, and 39 health posts are found in shalla district from which we selected 8 health posts from eight different localities of the district.

\section{Study design}

Multi centred health post based retrospective cohort study was conducted using the data of moderate acute malnourished children aged 6-59 months which were admitted to TSFP and treated in 8 health posts in Shala district between May 1, 2017, and August 30, 2017.

The study was conducted on 201 MAM children who had been treated using dry premixed supplementary food fortified corn soy blended (CSB++) and 201 moderate acute malnourished children who have been treated using Ready to Use Supplementary Food (RUSF). and both treatment food is readily available at study area.

\section{Source and study population}

\section{Source population}

All moderate malnourished children whose age is between 6 to 59 month in Shalla district who has been screened using MUAC from community and registered to take supplementary feeding treatment under targeted supplementary feeding programme.

\section{Study population}

All moderate malnourished children whose age is between 6 to 59 month in Shalla district screened and included to TSF programme between May 1, 2017 to August 30, 2017.

\section{Inclusion criteria and Exclusion criteria}

\section{Inclusion criteria}

Eligible 6-59 month age children who were screened from community using MUAC and identified to have MAM and admitted to TSF in selected 8 health posts between May 1, 2017, and August 30, 2017.

\section{Exclusion criteria}


Children identified from the community whose date of birth is missed by the mother.

Children screened and included in TSFP in other months of the year 2017.

\section{Sample size calculation}

The sample size was calculated based on the assumption that type I error $5 \%$, power of the study $80 \%$, proportion of recovery among MAM children used RUSF was 73 \% (exposed) and proportion of recovery among MAM children used CSB+ was $67 \%$ (unexposed) [16 ]. The sample size was calculated by using the formula Fleiss JL. 1981. pp.44-45. The minimum sample size required for each group is 122 . After adding $10 \%$ for incomplete records and multiplying with design effect 1.5 the final sample size was 402 .

\section{Sampling procedure}

In the study district, there are 39 health posts. Populations living around these health posts are assumed more or less homogenous. As the result, 8 health posts was selected at random using lottery method presuming that there were no information lost with the unselected health posts.

Also the SFP protocol for management of MAM works equally to all health post level. So in total, a sampling frame of children managed for MAM from 8 health posts in the district was prepared. Samples were allotted to each health institution using the probability proportional to size sampling. Finally, the children were selected by systematic random sampling from each institution based on their unique identification number.

Sampling frame was obtained from TSFP treatment registration book which documented by health extension worker work in the health post (figure 1).

\section{Data collection technique and procedure}

Data were collected by record review and interviewing using a semi structured questionnaire (additional file 1). Questionnaire developed based on published studies and adapted to local situation with certain modifications. the questionnaire was pretested in $10 \%$ of sample size to establish its ability to elicit relevant information, and necessary corrections were made on the questionnaire following the pretesting. The questionnaire was prepared in English and translated to Afaan oromoo because information on registration book is documented using Afaan oromoo in the health posts.

Data were collected by experienced 8 diploma nurses supervised by 2 Bsc nurse and data collector and supervisor was trained on how to collect quantitative and qualitative data.

The questionnaire included information on, individual factors affecting recovery time, health care related factor affecting recovery time collected by record review from selected health post, and family related factor affecting recovery time, Environmental related factor affecting recovery time data was collected by interviewing the MAM child family care giver. Data collection was completed in 10 days from January 110, 2018. 
Data quality assurance

Both the data collectors and the supervisor were given 1 days training regarding the objective content of the study to capacitate the skill of data collection methods.

As part of the training, the data collection tool was pretested at shalla district before the actual data collection is done to maintain data quality.

Completed questionnaires was collected on daily basis and checked for completeness and consistency by immediate supervisors. Data Cleaning was done on daily basis and timely feedback was communicated to the data collectors.

Incorrectly filled or miss ones was back to the respective data collectors for correction.

The principal investigator was supervised five percept of the secondary data collection and confirmed the data are correctly collected from the beneficiary registration and their care takers.

Ten percept of the data was re-entered in order to compare and assure the quality of the data.

\section{Study variables}

\section{Dependent variable}

Time to recovery of moderate acute malnutrition treatment.

\section{Independent variable}

Individual factor. care taker age, sex, place of residence, Admission weight, MUAC,WFH Z score category

Family factor: Educational status, Occupation and marital status of care giver, House hold food insecurity, inadequate care and feeding, Family income ,Child feeding practice .

Environmental factor: Drinking Water source, Safe solid disposal/stool disposal, un healthy household environment.

Health care related factor: Treatment foods, Follow up status, Health education topic covered by HEW, Food sharing, cooking demonstration conducted

\section{Operational definition}

Censored data (unobserved outcome): time-to-event is not observed.

Subjects are said to be censored if they are lost to follow up or drop out of the study, if the study ends before they recover or have an outcome of interest[3].

Defaulter: Child absent from the program for three consecutive visits and confirmed[5]. 
Intermittent follow up: Lost the TSFP program at least once during treatment period [25].

Non response: (Failure to respond to treatment) where child fails to reach the discharge criteria over a period of four months[25].

Overcrowded condition: Is a condition occurring when Person/room is greater than 2 person per 1 room, 3 person per 2 rooms, 5 person person 3 rooms.

Recovered: Children reach the target weight gain of $13 \%$ from admission weight and MUAC $\geq 12.5 \mathrm{~cm}$ or $\geq-2$ z-score period of two consecutive distributions[5].

Targeted Supplementary Feeding programme: Is the program which aims to rehabilitate children under five years old as well as pregnant and lactating women (PLW) identified as moderate acute malnutrition.

Time of recovery: Acceptable to stay the program with in less than or equal to sixteen weeks [5].

Transport access: Easy to reach desired distinction by means of a specific transport system.

Super cereal (CSB++): (Corn Soy Blend) a pre-mixed fortified blended food which contains Energy/Nutrient/100g, $787 \mathrm{kcal}, 33 \mathrm{~g}$ protein and $20 \mathrm{~g}$ fat.

\section{Data processing and analysis}

After the data were checked for its consistency and completeness, it was entered to EPIINFO version 7.0 then exported to SPSS version 20 for cleaning and analysis.

Summary statistics like frequencies, proportion were carried out to describe the socio-demographics data and other characteristics. Proportional of hazard rate is calculated to measure the association and the probability of time to recovery among MAM children during the specified period. Kaplan- Meier product limit, life table analysis and log rank test were used to estimate the time to recovery and the cumulative proportion surviving in agiven interval, and compare the survival curves, respectively. Cox proportional hazard regression was used to identify predictors of time to recovery.

Bivariate cox regression analysis was carried out, and independent variables with $p$-values of $\leq 0.25$ were included in multivariate cox regression analysis.

Prior to the multivariate analysis, multicollinearity diagnostics was performed, and there were no significant interactions between independent variables and some of highly correlated independent variable was removed.

Adjusted Hazard rate (AHR) with 95\% confidence intervals (Cls) was used as an effect measure. A pvalue of equal or less than 0.05 was considered as significant.

\section{Ethical clearance}


Ethical clearance was obtained from Institutional Review Board of Hawassa University College of Medicine and health Sciences. Official letters was taken from west Arsi zone Health department prevention control office, and consent was taken from individual care giver participated in the study.

\section{Data dissemination}

The final report was presented to Hawassa University College of Medicine and health Sciences. Copies were provided to west Arsi zone health office. It will be disseminated through publication of the findings, in peer reviewed reputable journal and presentations on scientific conferences.

\section{Results}

\section{Socio demographic characteristics of study population}

A total of 402 MAM children were included in the study from these female constituted $54.2 \%$. The median (IQR) ages of children was $18(12,24)$ month. The mean number of family members of the respondent households was $5.19 \pm 0.5$. Regarding Occupation of house hold leader $371(92.25 \%)$ of them are farmer and $19(4.75 \%)$ are private employee, $12(3 \%)$ are daily laborer.

Of the total 402 Sex of household leader $401(99.75 \%)$ are male and only $1(0.25 \%)$ are female and also about 237 (58.75\%) has transport access to nearest healthy facility and the rest $165(41.2 \%)$ of house hold had no transport access to distribution centre.

From all the children admitted to TSFP, the majority 394 (98\%) received Vitamin A,n99 (24.6\%) children had taken deworming and $336(77.5 \%)$ received measles vaccination.

Out of 402 children admitted to TSFP, 54(13.4\%) children were affected by diarrhoea, and the rest 347 $(86.7 \%)$ are not; only $2(0.5 \%)$ children were affected by cough with difficulty of breathing, and fever and the rest 398(99.8\%) are not affected.

Regarding the admission types of children to the TSFP in this study, 377 (93.75\%) children were newly admitted and 19 (4.8\%) were re-admission, and the remaining $6(1.5 \%)$ were return after default. Among the study cohort of children treated in the programme, 288 (71.6\%) was recovered, and $114(28.4 \%)$ were censored (table 1).

Among family related Factor affecting time recovery, the most (87.5\%) of the children started Complementary food at 6 month and the remaining 50 (12.5\%) children started complementary food at 5 month, about $396(98.5 \%)$ mother breast feed their children but the rest $6(1.5 \%)$ mother is not breasts fed their children for 2 year.

Out of total children data reviewed 330(82.2\%) beneficiary children of TSFP was in one Supplementary feeding Programme, and the remaining $72(17.75 \%)$ were supported under two or more Supplementary 
feeding programme. Likewise $160(40 \%)$ house hold are in other programme providing food support and the rest $242(60 \%)$ are only get food support from blanket supplementary feeding programme.

Regarding House hold food availability all time, of the total children family assessed, only $2(0.5 \%)$ of the child family have a food at all time, and $385(95.7 \%)$ of the child family were some times encounter food shortage and the rest 15(3.75\%) were mostly encounter food shortage in the house hold.

Out of all MAM child family interviewed $393(97.8 \%)$ of them get health education related to TSFP but Cooking demonstration is not conducted for 277 (68.9\%) care giver, the total number of care giver who know the exact amount of food ration given for children during food distribution was 382(95\%)and the rest 20(5\%) did not know.

Regarding supplementary food ration use for MAM children 226(56.2\%) care giver used the treatment food ration only for MAM diagnosed child. the rest $176(43.8 \%)$ of them is not. similarly, One hundred sixty (39.8\%) MAM children treated at TSFP were shared treatment food for other family members especially for Child of the house hold, and $16(4 \%)$ shared with parents/care giver. In respect to follow up status Three hundred fifty one (87.3\%) admitted children were attending TSFP ration distribution regularly and the rest $51(12.7 \%)$ of children were intermittently follow food ration distribution.

Concerning availability of latrine, $193(48 \%)$ of the households had latrine and 209(52\%)were had no latrine and use open field for defecation. In regard to solid waste disposal 356 (89\%) people dispose solid waste in open field and $46(11 \%)$ use solid waste disposal site.

Related to water source of the households in the study area 366 (91\%) getting water for drinking purposes from improved water source and the rest 36 (8.9\%) uses unimproved water source for the households. In addition about 307(76.4\%) house hold had enough water supply to drink and other purposes were as 95(23.6) had no enough water for the use of all purposes.

Regarding living condition of house hold environment 284(70.6\%) households of children admitted to TSFP were leave in overcrowded condition greater than two person per room and $118(29.3 \%)$ are not live in an overcrowded house.

\section{Proportion of recovery of MAM children at different week time interval during study period.}

The proportion of recovery of MAM children treated using TSFP in shalla district was $82.8 \%$ at 16 week with hazard rate of 0.26 , the majority of recovered children were found among those treated using RUSF is $(87.5 \%)$ where as those used CSB+ and oil were $(55.7 \%)$. The median (IQR) time to recovery was 15 $(13,18)$ week, and the average recovery time for children treated by RUSF was 13 weeks and those used $\mathrm{CSB}+$ and oil was 16 weeks.

There were a difference in recovery time among children who were treated by super cereals and CSB+ and oil. 
The study also showed that form 402 children admitted to the programme 5 children lost follow up for unclear reason and 59 of them remains not to recover within 16 week duration.

Each MAM children on treatment of TSFP in shalla district has the probability of recovery is $7.7 \%$ per person per week time. And the cumulative probability recovery from MAM after 1 month time on treatment is $27.4 \%$ (table 2 ).

\section{Predictors of time to recovery}

In this study the findings of bivariate analysis showed that person with primary child care responsibility, education status of house hold leader, number of house hold member, transport access to nearest TSFP distribution centres, comorbidities such as diarrhoea, fever, measles vaccination, admission MUAC ,admission status ,house hold food security status, open field disposal of wastes, treatment food share to other family members, regular follow up treatment food, types of treatment food, type of water source and being no enough water were significantly associated with dependent variable at $p$-value of $<0.25$, and can be considered as a candidate for multivariate regression analysis (table 3 ).

On the multivariable Cox-regression analysis, variables that have significant level at $95 \% \mathrm{Cl}$ and $\mathrm{p}$-value < 0.05 were considered to be the predictor of time to recovery of children with moderate acute malnutrition admitted at shalla district TSFP.

The multivariate cox regression analysis of this study showed that, transport access to nearest health facility, Admission MUAC, house hold food insecurity status, type of treatment food used and follow up status were a significant predictors of time to recovery of MAM treatment( table 4, Figure 2, Figure 3 ).

\section{Discussion}

The study assessed time to recovery from MAM and its predictors among children aged 6-59 months treated at targeted supplementary feeding program at Shala district. The mean time to recovery was 15 weeks. Transport access to nearest health facility, co morbidities such as diarrhoea, Admission MUAC, house hold food insecurity status, type of treatment food used and follow up status were identified as independent predictors of time to recover.

In this study the overall MAM treatment mean time to recovery was 15 week (SD, 3.3) which is consistent with Ethiopian national standard malnutrition treatment guide line which is $£ 16$ week [5]. Similarly, the studies conducted in sidama zone SNNPR Ethiopia, (CSB+=67\%, RUSF $=73 \%$ ) timely recovered at the end of 16 week [13] .This may be due presence of regular follow up by nongovernmental organization working on it and continuous health education support given by health extension service worker at their locality .

The study also found that the median (IQR) time to recovery was $15(13,18)$ weeks. Which is consistent with the Ethiopian national malnutrition treatment guide line recommendation 16 week [5] but this is lower than the study conducted in Jimma zone/Ethiopia [1]. 
The study showed recovery time of TSFP admitted children treated by super cereal was 13 weeks and CSB+ and oil was 16 weeks this shows that children treated by super cereal has lower median time to recovery than CSB+ and oil treatment groups, which implies children treated by super cereals was 2.4 times higher estimated better timely recovery than children treated by CSB+ treatment groups (AHR $=2.44,95 \% \mathrm{Cl}, 1.85-3.21)$ p-value $<0.05)$ which is consistent with study conducted in sidama zone/SNNP Ethiopia which showed the hazard rate for children in the CSB group was (AHR $=0.8595 \%$ Cl:0.73- 0.99), which indicated that they had $15 \%$ lower recovery . A recovery rate of children at the end of the 16 week treatment period is higher in the RUSF group (73\%) than in the CSB group (67\%) [13].

Similarly the other study conducted in Jimma /Ethiopia showed that MAM children treated by CSB+ and oil is (49.2\%) under TSFP was lower timely recovery than children treated by super cereals. From this we understood that treating MAM child with supercereal is more effective than CSB+ and oil.

Additionally, the study showed that child admission MUAC has also associated with time to recovery from MAM, child admitted with MUAC ${ }^{3} 11.6-12.5 \mathrm{~cm}$ has recover early within 16 week than child admitted with MUAC $=11.5 \mathrm{~cm}$ (AHR 1.18, 95\% Cl 1.13-1.23, p-value< 0.05$)$ which is consistent with a study conducted in Jimma showing children who has higher MUAC on admission 11.5-11.9 cm (AHR $0.4095 \% \mathrm{Cl} 0.31-0.52$ ) better recovered timely than child with $11.0-11.4 \mathrm{~cm}$ (AHR $0.2495 \% \mathrm{Cl} 0.16-$ $0.36) p<0.05$ [1].this indicates that early child screening for malnutrition and giving treatment enable as to get better outcome.

This study also revealed that transport access to the nearest health facility where targeted supplementary feeding service given was also significantly associated with the time to recovery. Among family who has no transport access, proportion of children recovered from MAM is (AHR $0.5095 \% \mathrm{Cl}, 0.38-0.64$ ) $p<0.05$ which is consistent with the study done at kamba district south Ethiopia [17]. This indicates that child who has transport accesses has got the service easily and show better prognosis.

The proportion of timely recovery among children from house hold with severe food insecure (AHR 0.49, $95 \% \mathrm{Cl} 0.21-0.51) \mathrm{p}<0.05$ which show $51 \%$ lower chance of recovery than moderate food insecure. likewise the study done in Jimma zone Ethiopia showed children from family with severe food insecure has $86 \%$ lower chance to recover timely than moderate food insecure (AHR $1.8695 \% \mathrm{Cl} 1.22-2.83$ ) $\mathrm{p}<0.05$. within 16 weeks[1].Another study conducted in Amahara region Awi zone showed the median recovery time was 62 days for children from food secure households $(95 \% \mathrm{Cl} 61.65-62.35)$ and 63 days for children from food insecure households $(95 \% \mathrm{Cl} 62.78-63.22)$ [12]. This indicates that Children from food secure households had better timely recovered than child from moderately food insecure house hold. This study also shown that MAM children admitted to TSFP that had regular treatment follow up every month had (AHR 1.48, 95\% Cl 1.09-2.00, p-value 0.01) 48\% higher proportion of recovery than intermittent treatment follows up. This may be related with appropriate use of treatment food and other health services. 


\section{Conclusions And Recommendation}

The median Time to recovery of most MAM children admitted to TSFP is less than 16 week. TSFP time to recovery was $82.8 \%$ at 16 week in shalla district. Predictor of time to recovery were, house hold food insecurity, intermittent treatment follow up, admission MUAC and type of treatment foods (RUSF and CSB+). There should be clear guidance on cut-offs for admission and discharge from MAM management programmes by considering the food security status of the locality.

Study on measuring effectiveness of treatment and efficacy of MAM treatment food should done.

Nutritional counselling on proper use of treatment food should be intensively done.

Further research should be done on the link between different morbidities and the effect of addressing these conditions on acute malnutrition.

\section{Limitation}

Food insecurity data is not collected in detail

Study was conducted in one district only.

Implication limited to immediate effect of the work station.

\section{Abbreviations}




\begin{tabular}{ll} 
AHR & Adjusted Hazard Ratio \\
\hline AM & Acute Malnutrition \\
\hline CMAM & Community Based Management of Acute Malnutrition \\
\hline CHR & Crude Hazard Ratio \\
\hline CSB + & Fortified Corn Soya Blend \\
\hline DMFASS & Disaster Management and Food Security Sector \\
\hline DPPA & Disaster Preparedness and Prevention Agency \\
\hline EOS & Emergency Out Reach Strategy \\
\hline EDHS & Ethiopian Demographic and Health Survey \\
\hline IQR & Inter quartile range \\
\hline MAM & Moderate Acute Malnutrition \\
\hline MUAC & Mid upper Arm Circumference \\
\hline OR & Odds Ratio \\
\hline RUSF & Ready to Use Supplementary Food \\
\hline SAM & Severe Acute Malnutrition \\
\hline SPSS & Statistical Package for Social Science \\
\hline TSFP & Targeted Supplementary Feeding programme \\
\hline UNICEF & United Nation's International Children's Emergency Fund \\
\hline WFP & World Food Programme \\
\hline WHZ & Weight for height Z-score \\
\hline WHO & World Health Organization \\
\hline OA
\end{tabular}

\section{Declarations}

\section{Ethics approval and consent to participate}

Ethical approval was received from institutional review board at the college of medicine and health science of Hawassa University.

\section{Consent for publication}

It is the retrospective study and the data was extracted from patient registration. 
All the data were included in the manuscript and the SPSS data is also uploaded in the supplementary material

\section{Competing interests}

No conflicts of interest prevail among researchers.

\section{Funding}

No fund

\section{Authors' contributions}

KK- participated in the design of the study and data extraction, performed statistical analysis, and drafted the first manuscript.

WT- participated in the design of the study, and reviewed the manuscript. All authors have read and approved the final manuscript

\section{Acknowledgements}

We would like to thank Hawassa University College of medicine and health sciences, school of public health for giving me a chance to conduct this research.

We would also express our gratefulness for west Arsi Zone health Department, for their helpful aid of the compulsory information, Shalla woreda health office for their genuine cooperation and facilitation to get appropriate information.

\section{References}


1. Philip J, Kate S, Mekitie W, Alemayehu A, Hanqi L, Benti G, Kiya K, Yilak G, Tefera B,

Paluku B. Children with Moderate Acute Malnutrition with No Access to Supplementary Feeding Programmes Experience High Rates of Deterioration and No Improvement. PLOS ONE I DOI:10.1371/journal.pone.0153530 April 21, 2016.

2. UNICEF / WHO / World Bank Group Joint Child Malnutrition Estimates. Levels and trends in child malnutrition. May, 2017

3. Reginald A. Annan L, Patrick W, Rebecca B. Management of Moderate Acute Malnutrition Current Knowledge and Practice. CMAM Forum. September, 2014.

4. Central Statistical Agency Ethiopia and ICF International Ethiopia. Demographic and Health Survey 2011. March, 2012.

5. Federal democratic republic of Ethiopia, Ministry of health. Guidelines for the management of acute malnutrition. July, 2016.

6. World Food Programme. Outcome evaluation study of targeted supplementary feeding program. University of Copenhagen; Copenhagen Denmark, Jimma University; Jimma, Ethiopia, Consultant for World Food Programme .June, 2009.

7. Disaster Risk Management and Food Security Sector, of the Ministry of Agriculture and Rural Development, Federal ministry of health of Ethiopia. Guidelines For the Management of Moderate Acute Malnutrition, in Ethiopia. September, 2012.

8. Robert A, Andrew C, Andrew B, Janet H, Helen B, Woods B, Lindsay B, Michelle H. Relationship between recovery and relapse, and default and repeated episode of default in the management of acute malnutrition in children in humanitarian emergencies

(Systemic review protocol). Oxfam GB for Oxfam International. April, 2016

9. Amsalu B. Ayana E. Tsedeke W. and Alemu S. Determinants of acute malnutrition among children aged 6-59 months in Public Hospitals, Oromia region.BMC Nutrition. November, 2015. 
10. A partnership study for the USAID ENGINE programme in Ethiopia, implemented

by Valid International, Jimma University and Save the Children. Outcomes of

Moderate Acute Malnutrition and their determinants in under-five children:

a prospective cohort study from a food-secure setting in rural Ethiopia. Mana and Denso district of Jimma zone. May, 2014.

11. The republic of Uganda Ministry of Health. Integrated Management of Acute Malnutrition Guidelines Kampala. February, 2010.

12 Alemu M, Nicola J, Belele T. Tackling child malnutrition in Ethiopia; Young lives project working paper No 19; Save the children UK 26. 2010.

13 Crystal K. Tina van d. Derek S. Stanley Z. Treatment of moderate acute malnutrition with readyto-use supplementary food results in higher overall recovery rates compared with a corn-soya blend in children in southern Ethiopia. The American journal of clinical nutrition.2012.

14 W/amilak A., Dube J., Mulunesh A. and Sahai B. Risk factors associated with poor health outcomes for children under the age of 5 with moderate acute malnutrition in rural fagita lekoma district, Awi Zone, Amhara,Ethiopia. May, 2017.

15 Gabriel N, Patricia M, Anne Christine A, Véronique J, Julie J, Henriette T. Recovery rate of children with moderate acute malnutrition treated with ready-to-use supplementary food or improved corn-soya blend (CSB+). Public Health Nutrition, Volume 19, Issue 2 pp. 363-370

February, 2016.

16 Tarun G,Juan P, Pena R,Harshpal S, Sachdev. Lipid based nutrient supplements for treatment of children (6 months to 59 months) with moderate acute malnutrition. A systematic review. PLOSONE| https://doi.org/10.1371/journal.pone.0182096. September 21, 2017.

17 Negash A, Sebelewengel L, Direslgne M. Recovery Rate and Determinants in Treatment of Children with Severe Acute Malnutrition using Outpatient Therapeutic Feeding Program in Kamba District, Ethiopia. J Nutr Disorders of an open access journal. March, 2015.

18 Ferew L, Tewoldeberhan D, Habtamu F, Emily M. CMAM rollout in Ethiopia. the 'way in' to scale up nutrition. Field Exchange 43, Ethiopia Government experiences. July, 2012. 
19 Lindsey L, Kerri W, Zulfiqar A. Management of Severe and Moderate Acute Malnutrition in Children. Centre for Global Child Health, The Hospital for Sick Children, Toronto, Canada. July, 2016.

20 Sebastian V, Christian B, Aditi K, Kenneth H. and SV Subramanian. The association of parental education with childhood under nutrition in low- and middle-income countries. International Journal of Epidemiology. May, 2017: 312-323

21 Mark M. Lora I. Indi T. Systematic review of vitamin A supplementation in the treatment of children with severe acute malnutrition. World Health Organization. February 2012.

22 Cindy Y. Chang I. Richard J. Wang C., Ken Maleta,Megan D/ Mark J. Children

Successfully Treated for Moderate Acute Malnutrition Remain at Risk for Malnutrition and Death in the Subsequent Year after Recovery. J. Nutr. 143: 215-220, 2013.

23 Zottarelli L, sunil T. Influence of parental socioeconomic factor on stunting in Children less than 5 years Egypt. Eastern Mediterranean health journal October, 2010.

24 Kebede M. Kassahun A. Bikes D. Prevalence of Malnutrition and Associated Factors Among Children Aged 6-59 Months at Hidabu Abote District, North Shewa, Oromia Regional State. Open Access Journals, July 25, 2013

25 Global Nutrition Cluster MAM Task force. Moderate acute malnutrition decision tool for emergencies. June, 2014.

26 Zemenu y, Tsigereda B,Alemu M, Mesfin T, Sintayehu Y. malnutrition and associated factor among under five children(6-59) month at shashemene referral hospital west Arsi zone, oromia, Ethiopia. Allied Academies. January, 2016.

27 West Arsi zone health office performance plan,oromia,Ethiopia, september,2017

\section{Tables}

Due to technical limitations the tables are available as a download in the Supplementary Files.

\section{Additional File Legends}

Additional file 1 - Questionnaire to assess time to recovery to moderate acute malnutrition and its predictors among 6-59 months children targeted for supplementary feeding study primary data collection questionnaire.

Additional file 2 - Figure 1. The schematic representation of sample selection procedure in Shala district TSFP, January, 2018.

Additional file 3- Figure 2. A cumulative probability curve showing probability of recovery at the end of each week, shala District TSFP January 2018. 
Additional File 4- Figure 3. Kaplan Meier curve showing cumulative proportion staying in a programme among MAM children using different treatment food at end of each week shalla district TSFP, January 2018.

\section{Figures}

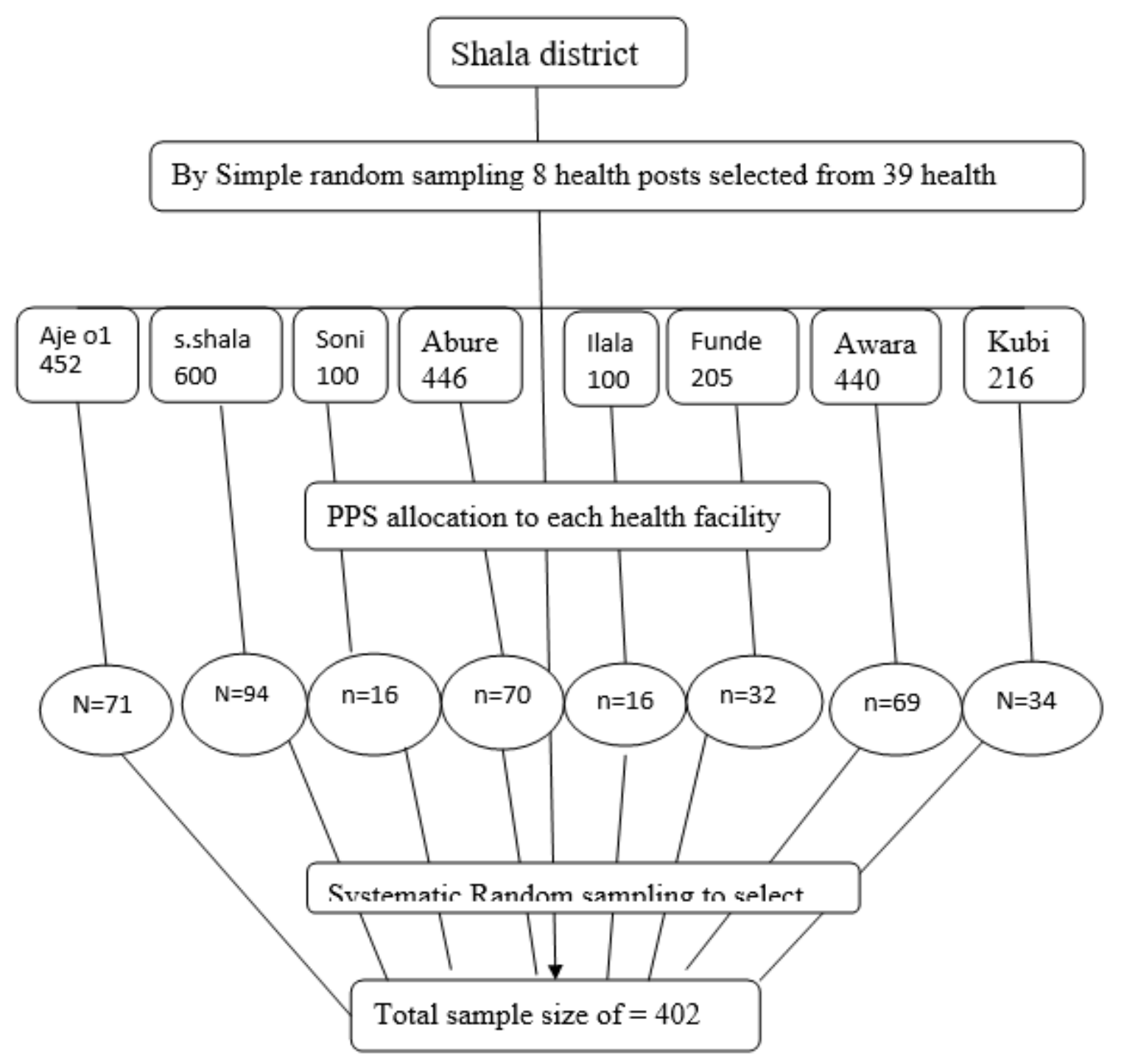

Figure 1

The schematic representation of sample selection procedure in Shalla district TSFP, January, 2018. 


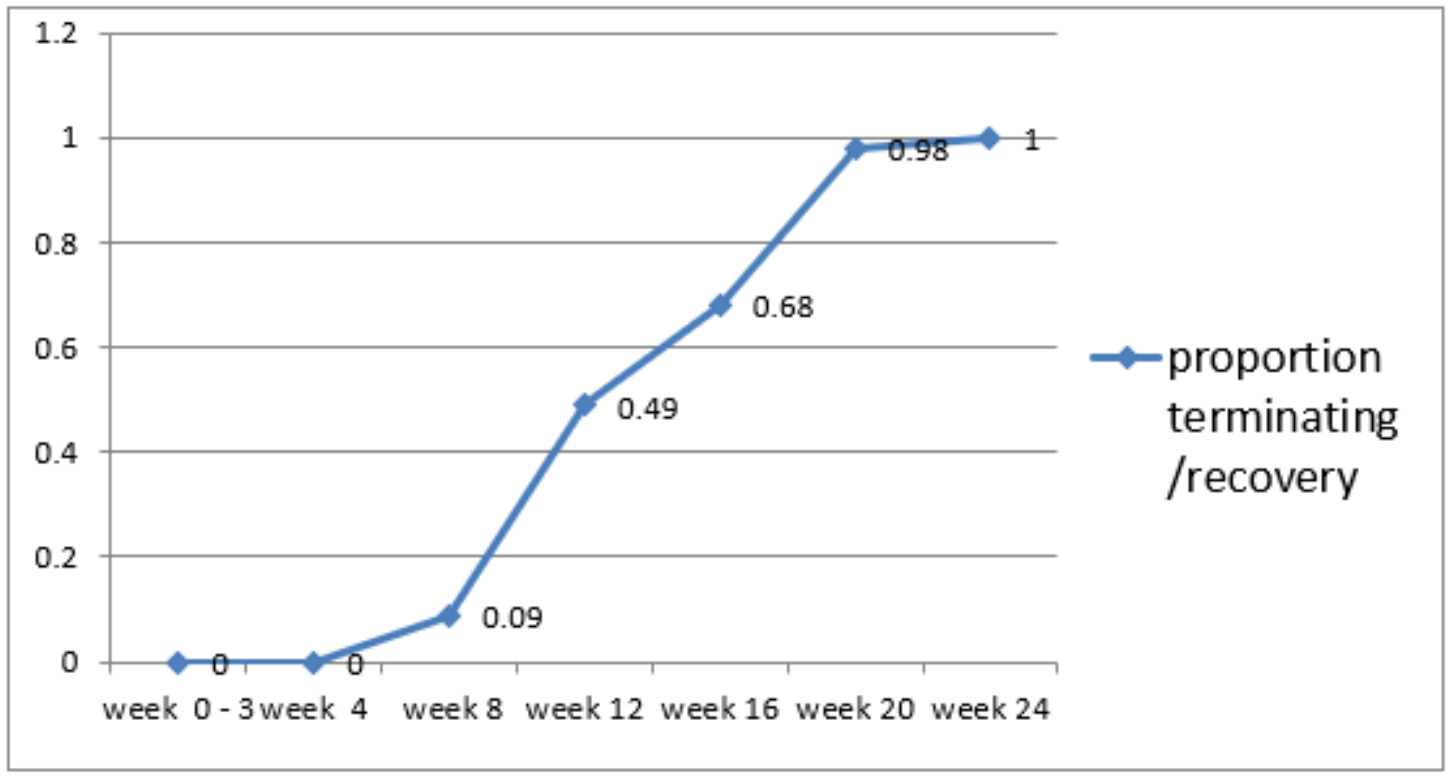

\section{Figure 2}

A cumulative curve showing probability of recovery at the end of each week, shala District TSFP January 2018

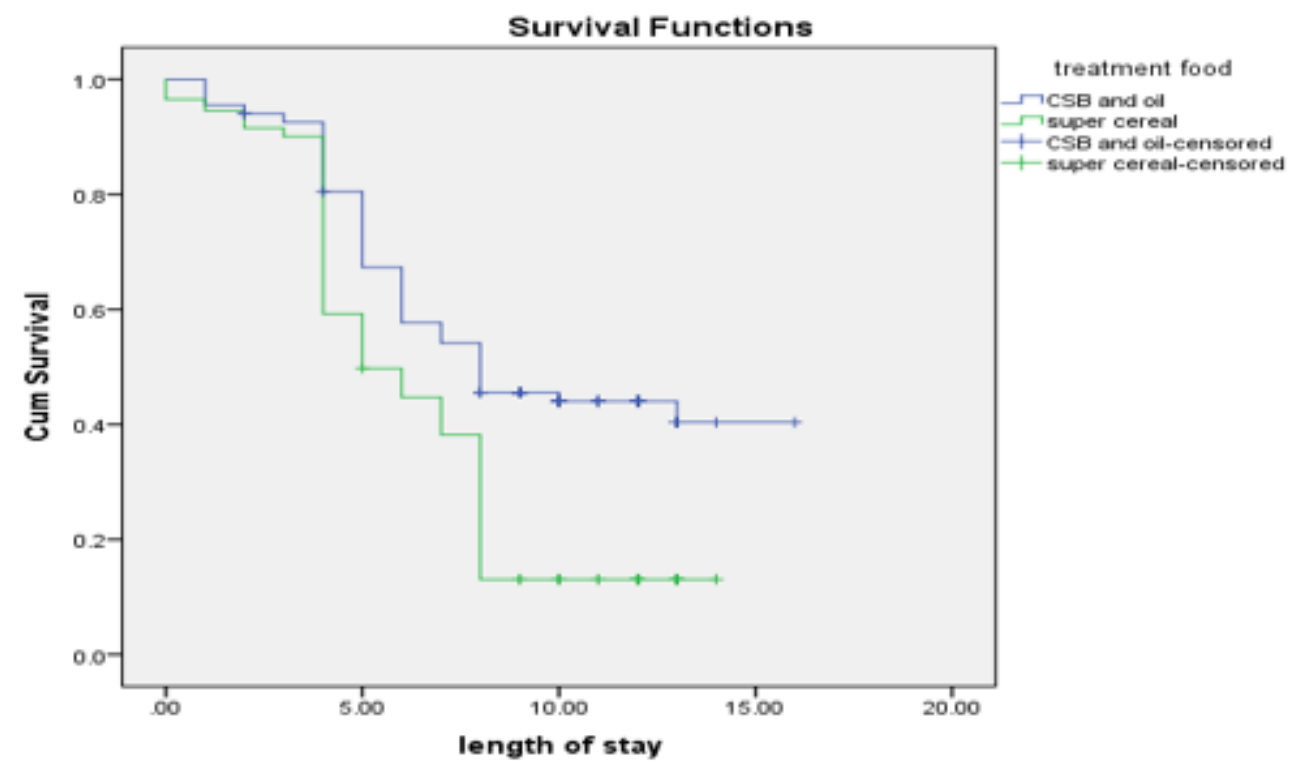

Figure 3

Kaplan Meier curve showing cumulative proportion staying in a programme among MAM children using different treatment food at end of each week shalla district TSFP, January 2018.

\section{Supplementary Files}

This is a list of supplementary files associated with this preprint. Click to download. 
- additionalfile23456figureHMXL.xml

- additionalfile1.pdf

- BMCtable.xml

- entereddatarecodedandreadyforanalysis.sav

- additionalfile.docx 